Extended Abstract

\title{
Transdimensional relations at the bases of music, symmetry, language, logic and information
}

\author{
Alexander Koblyakov \\ Moscow P.I. Tchaikovsky Conservatory, Russia, 125009, Moscow, Bolshaya Nikitskaya ,13 \\ E-Mail: akoblyakov@list.ru
}

Tel.: +7-903-240-0910

Accepted:

\section{Introduction}

A new approach to the analysis of a musical piece has revealed new types of relations, which we refer to as "transdimensional relations", or "transdimensionalism" (abbreviated TD) [1].

Transdimensionalism refers to the entire set of relations between spaces of differing dimensions within a multidimensional whole. ("Dimension" is the number of degrees of freedom, which allow both geometric and parametric interpretations).

Therefore, within a musical whole (a musical piece) the melodic plain (1-dimensional, 1D) is connected with 2-dimensional intervals (2D) and 3-dimensional chords (3D). An important aspect of transdimensionalism is a transition from one space to another, which can be referred to as a transdimensional transition (abbreviated TDt), using our terminology [2]. The entire history of music is a chain of such transitions: from single voice monody to two voice polyphony, to 2-dimensional intervals (X century), from 2-dimensional intervals to 3-dimensional chords-triads (XVII century), then from chords to poly-chords and poly-harmonies during the XX century. Let us look at a special case of transdimensional relations - "polydimensions" (abbreviated PD), meaning an object, which belongs to spaces of different dimensions. We will show that polydimensionalism lies at the base of music. We can look at the basic cell in modal music - the relationship between a stable tone and an unstable tone.

\section{Main Part}

The stable tone is independent from the unstable one, it is defined unambiguously, and has one degree of freedom. Whereas the unstable tone is dependent on the stable one and has two degrees of 
freedom: 1) it can resolve into stable tones, or 2) it can move away from it. From the point of view of musical grammar, the unstable tone is unambiguous like the stable tone (music as language). Whereas from a contextual meaning, the unstable tone is bimodal (music as speech), and is 2-dimensional.

Therefore, the pair "stable tone-unstable tone" is polydimensional, one of its parts is 1- dimensional, while the other is 2-dimensional ( $P D=1 \mathrm{D}: 2 \mathrm{D}$ ). The polydimension 1D:2D is the primary basic cell of transdimensionalism, which allows one to separate contextual meanings from non-contextual meanings.

The link between music and symmetry is well known. Transdimensional relations and polydimensionalism allow one to consider the pair of "symmetry-asymmetry" from a different perspective. Symmetry is the unity of preservation and change. Transdimensionalism expands the very term "change": a change in the same dimension of musical space is now complimented by the change in the dimension of that space (transdimensional transition). Another attribute of symmetry is "preservation", which also becomes more complex. Polydimesionalism expands the term "invariance", since now one is taking into account the contextual links of an object (a single melody, removed from its polyphonic or harmonic context, is simultaneously "the same" and "different"!). The connection of transdimensionalism and symmetry ("transdimensional symmetry") allows us to see a new quality (parameter) that appears during the formation of the whole.

Let us look at the same pair, "stable sound-unstable sound" in the context of language. Here it is also polydimensional. On the one hand, both units in the pair are equal and symmetrical, differentiated only by the plus and minus signs (or by "color"). On the other, the word "un" (negation) creates a new quality (or parameter): not only the negation of a statement ("stable sound"), but also an unbreakable bond with it (since we are talking about negation not in the abstract, but of a specific statement). As a result, we find that negation is a complex unit of the pair, as apposed to the simple statement. From this point of view, the pair is polydimensional ( $P D=1 \mathrm{D}: 2 \mathrm{D})$ and asymmetrical. Therefore, all of the pairs, which are associated with negation, are polydimensional and asymmetrical (truth-untruth, to benot to be, etc.). The second units (context dependent) of these dyads form a pair that is a "linkage" with the first units (context independent). The pair "symmetry-asymmetry" is also polydimensional!

It is common knowledge that our thinking is based on binary oppositions, which are considered as symmetrical. As it turns out, oppositions can be simple and complex (context dependent), and complex oppositions are asymmetrical! This includes not only pairs with negative, but also with reflexive units (object-subject, action-observation), a secondary action (stimulus-reaction, vibrator-resonator), synthesis inside one of its parts (horse-rider), etc.

All of these are examples of PD of the type 1D:2D or "transdimensional asymmetry". From this we get several important logical consequences. Firstly, context-independent terms are separated from context dependent ones; in particular, we find the polydimensionality (which was not so obviously till now) of logical pairs such as "yes-no", "true-false", etc. Secondly, when one takes into account transdimensional relations and polydimensionality, classical paradoxes cease to be paradoxical. For example, the barber paradox (Bertrand Russell): all inhabitants of the village are context-independent (1-dimensional), while the barber is polydimensional, $\mathrm{PD}=1 \mathrm{D}: 2 \mathrm{D}$. The king's order does not take this into account, thereby creating a paradox! (We can also consider other paradoxes such as "I am lying" or "I am asleep", etc.).

Here arises the possibility of creating a new type of logic - the "logic of creativity"-, which integrates classical and non classical logic into a single whole through the use of $P D=1 D: 2 D$ 
Let's move on to information theory, the basic cell of which is a "bit", that is the same pair as "yesno", or 0 and 1. It was previously thought that the members of the pair are equal and symmetrical. However, this is only partly true. The negation "no" forms a "linkage state" with the affirmation, which justifies the transition to Q-bits. Let us remember, that the idea of Q-bits came from the physical sciences (quantum mechanics). We arrived at the idea from studying the humanities, which underscores the universal nature of these new relations.

\section{References}

1. Koblyakov A. Semantic aspects of self-similarity in music. Symmetry: Culture and Science, 1995 , v. 6, number 2, p. 297-301

2. Koblyakov A. Theorem of transdimensional transition. Works of the International conference “Mathematics. Computer. Education”, Moscow-Dubna, 2000, p. 22-42 (In Russian)

(C) 2015 by the authors; licensee MDPI and ISIS. This abstract is distributed under the terms and conditions of the Creative Commons Attribution license. 